\title{
Mechanism for the occurrence of paramagnetic planes within magnetically ordered cerium systems
}

\author{
Nicholas Kioussis, ${ }^{*}$ Bernard R. Cooper, and Amitava Banerjea ${ }^{\dagger}$ \\ Department of Physics, West Virginia University, Morgantown, West Virginia 26506 \\ (Received 24 August 1987; revised manuscript received 23 May 1988)
}

\begin{abstract}
Hybridization of moderately delocalized $f$ electrons with band electrons gives rise to a highly anisotropic two-ion interaction. Previously it has been shown that such an interaction explains the experimentally observed unusual magnetic behavior of $\mathrm{CeBi}$, yielding a phase transition from a higher-temperature type-I $(\uparrow \downarrow)$ to a lower-temperature type-IA $(\uparrow \uparrow \downarrow \downarrow)$ antiferromagnetic structure. If the hybridization-mediated interaction is the key to understanding the magnetic behavior of such moderately delocalized $f$-electron systems, we should expect to be able to understand on this basis the even more unusual magnetic behavior of $\mathrm{CeSb}$. In $\mathrm{CeSb}$, there is a sequence of magnetic structures in which the higher-temperature structures involve a periodic stacking of paramagnetic $\{001\}$ planes alternating with magnetically ordered $\{001\}$ planes of [001]-moment alignment. In this paper we show that such a coexistence of paramagnetic and magnetically ordered $\mathrm{Ce}^{3+}$ sites can be understood on the basis of the hybridization-mediated interionic interaction when there are cubic crystal-field (CF) interactions of comparable strength. The tendency to form paramagnetic planes is found to increase with increasing CF strength ( $\Gamma_{7}$ ground state); and the stability of the up-down paramagnetic plane arrangement at high temperatures is shown to arise from the reconciliation of the magnetic ordering with the $\mathrm{CF}$ interactions. We also find that for a certain range of parameters a different novel situation occurs, with a fully nonmagnetic (singlet) ground state for the $\mathrm{Ce}^{3+}$ ion. This singlet state is not Kondo-like, and occurs in such a way that the system would be expected to fluctuate between two differently polarized states, one of which is the singlet state.
\end{abstract}

\section{INTRODUCTION}

Among cerium compounds, the $\mathrm{Ce}$ monopnictides, $\mathrm{Ce} X(X=\mathrm{N}, \mathrm{P}, \mathrm{As}, \mathrm{Sb}, \mathrm{Bi})$, provide a class of anomalous compounds with highly unusual magnetic properties. ${ }^{1-3}$ In particular, the heavier monopnictides, $\mathrm{CeSb}$ and $\mathrm{CeBi}$, are characterized by a very large magnetic anisotropy along the cube-edge direction of the $\mathrm{NaCl}$ lattice, ${ }^{4,5}$ a large reduction of the crystal-field (CF) splitting from the value expected from the point-charge model, ${ }^{6}$ a complex magnetic phase diagram containing unusual magnetic structures, ${ }^{7-9}$ and, finally, unusual magnetic excitation spectra. ${ }^{10-12}$ These anomalous features of Ce monopnictides are, moreover, distinct from those often found in cerium compounds, and which are loosely characterized as originating from an interaction of $f$ electrons with $5 d$ conduction electrons giving rise to the so-called "Kondo-like" ( $f$-moment screening) behavior. ${ }^{2}$

The large cube-edge anisotropy and consequent magnetic structural behavior were successfully explained by Siemann and Cooper ${ }^{13}$ as arising from the hybridization of moderately delocalized $f$ levels with band electrons via a mixing mechanism originally introduced by Coqblin and Schrieffer (CS) (Ref. 14) for dilute cerium alloys. The joint hybridization of a pair of $\mathrm{Ce}^{3+}$ ions with band electrons gives rise to a highly anisotropic interionic interaction which is generically related to the RudermanKittel-Kasuya-Yosida (RKKY) interaction, ${ }^{15}$ but where the interaction with the band electrons is through the orbital rather than the spin part of the $f$-electron moment. ${ }^{16}$ The theory accounts well for the main features of the anisotropic magnetic equilibrium ${ }^{3,13}$ and excitation $^{17,18}$ behavior of $\mathrm{CeBi}$, reproducing ${ }^{13}$ the first-order transition from the high-temperature type-I antiferromagnetic (AF-I) structure $(\uparrow \downarrow)$ to the low-temperature type-IA antiferromagnetic (AF-IA) structure $(\uparrow \uparrow \downarrow \downarrow)$.

Among the cerium monopnictides, $\mathrm{CeSb}$ exhibits the most complex phase diagram, containing at least 14 different magnetic structures for $H<70 \mathrm{kOe} .^{2,8,9} \mathrm{CeSb}$ orders with a first-order transition $a^{2} T_{N} \simeq 16.2 \mathrm{~K}$ and undergoes six additional first-order phase transitions in zero field. ${ }^{2}$ The high-temperature phases $(>8.5 \mathrm{~K}$ ) (referred to as AFP here) are commensurate with the lattice and correspond to a periodic stacking of nonmagnetically ordered $(P)$ and ferromagnetic $(\boldsymbol{M})(001)$ planes with an up or down magnetization along the [001] direction and a close-to-saturation moment $\left.\left(\sim 2.1 \mu_{B}\right)\right)^{8,9}$ In zero field, the nonmagnetic planes are isolated, i.e., they always have neighboring up and down ferromagnetic planes, and the distance between the $P$ layers increases with decreasing temperature. Below $T_{0} \sim T_{N} / 2=8.5 \mathrm{~K}$ the nonmagnetic planes disappear, giving a type-IA phase $(\uparrow \uparrow \downarrow \downarrow)$. This is actually never fully established, because the wave vector $\mathbf{q}=(2 \pi / a)(0,0, q)$ is strongly sample dependent and varies ${ }^{12}$ from $q=0.51$ to 0.503 .

In $\mathrm{CeSb}$, the octahedral crystal field splits the $\mathrm{Ce}^{3+}$ free-ion $J=\frac{5}{2}$ multiplet into a $\Gamma_{7}$ doublet and a $\Gamma_{8}$ quartet, with a CF splitting, $\Delta_{\mathrm{CF}}$, of about $37 \mathrm{~K}$ in the paramagnetic phase. ${ }^{20}$ Thus the strength of the two-ion (exchange) interactions in CeSb, as measured by the Neel temperature, is of comparable order of magnitude to the CF splitting; and consequently there is a strong competi- 
tion between hybridization-mediated two-ion interactions and crystal-field effects. (On the other hand, for $\mathrm{CeBi}$ the small CF splitting of about $8 \mathrm{~K}$, compared to the ordering temperature of $25 \mathrm{~K}$, indicates that the exchange interaction dominates the CF interaction.) Transport measurements $^{21}$ on $\mathrm{CeSb}$, and magnetization and neutron scattering measurements ${ }^{19}$ on $\mathrm{CeSb}_{1-c} \mathrm{Te}_{c}$ solid solution have clearly established that the CF ground state in $\mathrm{CeSb}$ in the paramagnetic phase is the $\Gamma_{7}$ doublet, even though previous polarized neutron scattering experiments ${ }^{22}$ were interpreted as yielding a $\Gamma_{8}$ ground state. As far as the nature of the nonmagnetic planes is concerned, i.e., whether these layers contain cerium ions in paramaganetic (Kramers doublet) or Kondo (singlet) states, inelastic neutron scattering studies ${ }^{19}$ of the magnetic excitation spectra at high temperatures $(14 \mathrm{~K})$ strongly support the view that the nonmagnetic planes are actually paramagnetic planes with the same level structure as in the paramagnetic phase, but with a larger CF splitting of about $43 \mathrm{~K}$. Specific-heat measurements ${ }^{23}$ on CeSb provide further confirmation of the para-nature (containing $\mathrm{Ce}^{3+}$ ions in Kramers doublet states) of the nonmagnetic layers, yielding an entropy value of $k_{B} \ln 2$ for $\mathrm{Ce}^{3+}$ ions in nonmagnetic planes. Moreover, resistivity measurements ${ }^{24}$ on $\mathrm{Ce}_{c}\left(\mathrm{La}_{0.76} \mathrm{Y}_{0.24}\right)_{1-c} \mathrm{Sb}$ have shown that the effect of diluting the $\mathrm{Ce}$ in $\mathrm{CeSb}$ with nonmagnetic $\left(\mathrm{La}_{0.76} \mathrm{Y}_{0.24}\right)$ (keeping the lattice constant unchanged), is to suppress the negative temperature slope anomaly of the more concentrated samples above the ordering temperature. This result contrasts with the rather simple picture of a Kondo effect in cerium monopnictides.

In spite of the large amount of experimental data available for CeSb, only a few theoretical attempts $\mathrm{s}^{25-28}$ have been made to explain, even qualitatively, its complex magnetic phase diagram and especially the coexistence of magnetically ordered and nonmagnetically ordered planes, a unique feature. A preliminary discussion, along the lines of the present work, involving competition between the two-ion anisotropic interaction, crystal-field effects, and distortional effects is given in Refs. 3 and 13. Theoretical calculations ${ }^{25-17}$ based on a phenomenological one-dimensional spin- $\frac{1}{2}$ anisotropic Ising model with competing nearest- and next-nearest-neighbor interactions examined the zero-field phase diagram. However, these theories failed to yield magnetic structures containing nonmagnetic planes. Takahashi and Kasuya, ${ }^{28}$ in a theory based on the $p$ - $f$ mixing model, examined the zero-temperature ground-state energies of various magnetically ordered structures in $\mathrm{CeBi}$ and $\mathrm{CeSb}$. In that work, the small difference in energy $(0.25 \mathrm{meV})$ between the AF-IA and $(\uparrow \uparrow \downarrow P)$ structures in CeSb indicates the possibility of coexistence of the nonmagnetic and ferromagnetic planes. However, at nonzero temperatures, assuming an entropy contribution to the free energy of $0.6 k \ln 2$ (Kondo singlet) from the nonmagnetic cerium sites, they find the rather unrealistic result that the $\uparrow \uparrow \downarrow P$ structure becomes more stable than the AF-IA structure only at temperatures above $26 \mathrm{~K}$. (The experimental ${ }^{2}$ Néel temperature of CeSb is $16.2 \mathrm{~K}$.)

The aim of the present paper is not to fully reproduce the exotic phase diagram of $\mathrm{CeSb}$, but rather to address the question of whether one can understand the main features of the magnetic equilibrium behavior of $\mathrm{CeSb}$, i.e., the coexistence of magnetic and nonmagnetic layers at higher temperatures, within the framework of the previously developed ${ }^{3,13}$ model. This takes into account the competing effects of the crystal-field and hybridizationmediated two-ion exchange interactions, and does not invoke an additional proposed ${ }^{28}$ mechanism, namely the effective suppression of the local $\mathrm{Ce}^{3+}$ magnetic moment due to Kondo spin screening. (This last process would give rise to ${ }^{28}$ a "superdense Kondo behavior.") In Sec. II we briefly review the model of hybridization-mediated interionic coupling in the $f^{1}$ system (which has been presented in detail elsewhere ${ }^{3}$ ). The effect of the anisotropic hybridization-mediated interionic interactions on the formation of Kramers doublets at the paramagnetic sites in the absence of a crystal field is discussed in Sec. III A. We then show in Sec. III B how a CF interaction, comparable in strength to the hybridization-mediated interionic interaction, helps to stabilize various magnetic structures containing paramagnetic layers in the hightemperature regime. Numerical results for the temperature dependence of the free energies of various magnetic structures, the transitions between these structures, and the variation of magnetization with temperature are also presented in Sec. III B and compared with experiment. We also discuss in that section how the large reduction of the $\mathrm{Ce}^{3+}$ local moment due to the CF effects (off-diagonal admixture) can be counteracted through the introduction of a weak distortional term in the Hamiltonian. The two-ion hybridization-mediated-interaction-induced dressing ${ }^{1,13}$ of the states of the paramagnetic sites, and the resultant enhancement of the CF splitting in the ordered phase relative to its "bare" value in the paramagnetic phase, as experimentally observed, are discussed in Sec. IV. The question of whether the presence of sufficiently strong $C F$ interactions relative to the hybridization-mediated exchange interactions could give rise to a ground state which is fully paramagnetic (i.e., with all the $\mathrm{Ce}^{3+}$ states being Kramers doublets) is investigated in Sec. V. We also discuss in this section the occurrence of a new peculiar type of fully nonmagnetic (singlet) ground state, which, however, is not Kondo-like. Finally, a brief summary and statement of conclusions are presented in Sec. VI.

\section{THE MODEL}

The treatment of hybridization between the quasilocalized $f$ electrons and band electrons was first developed by Coqblin and Schrieffer ${ }^{14}$ for dilute cerium alloys, and was later extended by Siemann and Cooper ${ }^{3,13}$ to develop the hybridization-mediated anisotropic two-ion interaction in cerium compounds. Here we briefly review the model. Starting with the Anderson model $^{29}$ for the singleimpurity problem, upon applying the Schrieffer-Wolf ${ }^{30}$ transformation, Coqblin and Schrieffer obtained an effective Hamiltonian which represents the band- $f$ hybridization in terms of resonant scattering. When this scattering Hamiltonian is treated in second-order perturbation theory, one obtains an interionic interaction, where the information between ions is transmitted by the 
scattered band electrons, as in the RKKY interaction, ${ }^{15}$ although in this case the interactions are highly anisotropic. In the $f^{1}$ case Cooper and Siemann ${ }^{3}$ have shown that the predominant two-ion coupling arises from the $m_{l}=0$ (with respect to the interionic axis) part of the quasilocalized $f$ wave function, corresponding to the piling up of charge along the interionic axis and hence to preference for moment alignment perpendicular to the interionic axis. (This result is true as long as the mixing potential is spherically symmetric for free-electron-like bands. ${ }^{3}$ Calculations ${ }^{31}$ based on a realistic band structure support this picture.) However, compromise forced by the different directions of interionic axes may lead to complex magnetic structures.

Upon transforming the wave functions $\left|J M_{J}\right\rangle$ for the $\mathrm{Ce}^{3+}$ ions from many "bonding axis" coordinate systems to one common crystalline coordinate system, the hybridization-mediated interaction Hamiltonian takes the form ${ }^{3,13}$

$\mathscr{H}_{\mathrm{CS}}=-\sum_{i, j} E_{i j} \sum_{\substack{\mu, v \\ \epsilon, \sigma}} B_{\mu \nu}^{\epsilon \sigma}\left(\theta_{i j}\right) e^{i(\mu-v+\epsilon-\sigma) \phi} L_{\mu \nu}^{(i)} L_{\epsilon \sigma}^{(j)}$,

where $i$ and $j$ label cerium sites, $\theta_{i j}$ and $\phi_{i j}$ are the angular coordinates of the interionic axis $\mathbf{R}_{i j}$ with respect to the axis of quantization chosen along the [001] direction in the crystal, and $\mu, v, \epsilon, \sigma$ label the single-ion states of the $z$ component $M_{J}$ of the angular momentum $\left(J=\frac{5}{2}\right)$ quantized along [001]. The standard basis (transition) operators, ${ }^{32} L_{\mu \nu}^{(i)} \equiv|\mu\rangle\langle\nu|$, transfer the $\mathrm{Ce}^{3+}$ ion on site $i$ from state $|\nu\rangle$ to state $|\mu\rangle$, the function $B_{\mu \nu}^{\epsilon \sigma}\left(\theta_{i j}\right)$ is given in terms of the matrix elements of the $J$ th $\left(\frac{5}{2}\right.$ th $)$ irreducible representation of the full rotation group, ${ }^{33}$ and finally $E_{i j}$ are phenomenological interaction range parameters, with $E_{n}$ giving the interaction strength with the $n$th nearest neighbor. ${ }^{3,13}$

In addition to the hybridization-mediated interionic interaction in Eq. (1), the quasilocalized $f$ electrons are subjected to a cubic crystalline electric field of the form ${ }^{34}$

$$
\mathscr{H}_{\mathrm{CF}}=B_{4} \sum_{i} \sum_{\mu, v}\left\langle\mu\left|\left[O_{4}^{0}+5 O_{4}^{4}\right]\right| v\right\rangle L_{\mu v}^{(i)},
$$

where $\left\langle\mu\left|O_{n}^{m}\right| v\right\rangle$ denotes the matrix element of the Stevens ${ }^{35}$ operator, $O_{n}^{m}$, between the single-ion states $|\mu\rangle$ and $|v\rangle$ of $J^{z}$; and the CF interaction parameter, $B_{4}$, is treated phenomenologically. For $f^{1}$ systems $\left(J=\frac{5}{2}\right)$, the CF splitting, $\Delta_{\mathrm{CF}}$, between the $\Gamma_{7}$ doublet (ground state for positive $B_{4}$ ) and the $\Gamma_{8}$ quartet is given by $\Delta_{\mathrm{CF}}=360 B_{4}$.

To study the equilibrium magnetic behavior of a system with the Hamiltonian of Eqs. (1) and (2), we perform a molecular field (MF) calculation. The thermodynamic behavior of the different magnetic structures is determined as follows. First, we feed in trial MF states, $|m\rangle$ of energy $\varepsilon_{m}$, (lower-case Latin italic letters denote MF states), for each sublattice of the structure (assuming that all moments are identical within a given sublattice) so that

$$
|m\rangle=\sum_{\mu} x_{\mu, m}|\mu\rangle .
$$

We then determine the thermal average of the transition operators from the equation

$$
\left\langle L_{\mu v}\right\rangle=\sum_{n}\left\langle n\left|L_{\mu v}\right| n\right\rangle f_{n},
$$

where

$$
f_{n}=\exp \left(-\epsilon_{n} / k_{B} T\right) / \sum_{m} \exp \left(-\varepsilon_{m} / k_{B} T\right),
$$

is the thermal occupation of the $n$th MF state.

Upon treating the two-ion interaction in Eq. (1) within the MF approximation, and subsequently diagonalizing the sum of the resultant expression and the single-ion CF term in Eq. (2), one finds a new set of $(2 J+1)-\mathrm{MF}$ eigenenergies, $\varepsilon_{m}$, and corresponding $\mathrm{MF}$ eigenstates, $|m\rangle$, for each sublattice. This procedure is repeated iteratively until one attains self-consistency. Usually, self-consistency is obtained after only few iterations, except for temperatures close to the ordering temperature where the convergence is slower and a few hundred iterations are needed.

\section{NUMERICAL RESULTS FOR COEXISTENCE OF PARAMAGNETIC AND MAGNETICALLY ORDERED PLANES IN CERIUM COMPOUNDS}

In this section it will be shown that the main features of the unusual phase diagram of CeSb can be understood in terms of a theoretical model which takes into account two interactions that compete and that are comparable in magnitude, i.e., the hybridization-mediated two-ion interactions and the crystal-field interactions. These main features are the occurrence of magnetic structures in the high-temperature regime containing both magnetically ordered and nonmagnetic sites, and the transition at lower temperatures to structures containing solely ordered sites. The hybridization-mediated interionic interaction tends energetically to favor the magnetically ordered sites with local moments along $\langle 001\rangle$ and close to saturation. ${ }^{3,13}$ On the other hand, as will be illustrated in detail below, the crystal-field interaction (favoring $\langle 111\rangle$ moment alignment ${ }^{5}$ ) tends to favor the nonmagnetic sites, which being paramagnetic (Kramers doublets) in nature, do have a clear entropy advantage over the ordered sites at higher temperatures. In addition, the presence of crystal-field interactions introduces off-diagonal mixing between the free-ion states $|\mu\rangle$, and this reduces the local moments on the ordered $\mathrm{Ce}^{3+}$ sites. However, as will be shown in Sec. III B, upon introducing a small distortional term in the Hamiltonian, which tends to increase the diagonal matrix elements, one can restore the local moments to their saturated values. Thus the coexistence of magnetic and nonmagnetic sites represents a sort of compromise between crystal-field, distortional, and hybridization-mediated anisotropic interactions. In Sec. III A we first discuss the effect of the anisotropic hybridization-mediated two-ion interaction on the formation of Kramers doublets at the paramagnetic sites in the AFP 1 magnetic structure in the absence of CF interactions; we then discuss how the anisotropic two-ion interaction determines the free-energy contribution of the ordered sites in the AFP1 phase. This enables us to dis- 
cuss the expected temperature dependence of the magnetic structural behavior in the absence of crystal-field effects. Then, in Sec. III B, we investigate the role the crystal field plays in helping to stabilize various magnetic structures containing paramagnetic layers in the hightemperature part of the magnetically ordered regime.

\section{A. Effect of two-ion interactions on paramagnetic and ordered sites}

The various low-field and high-temperature $\left(T_{N} / 2<T<T_{N}\right)$ magnetic structures in CeSb contain the up $(\uparrow)$, down $(\downarrow)$, paramagnetic $(P)$ sequence of $(001)$ planes (AFP1) as the basic magnetic block unit. ${ }^{2,8,9}$ The paramagnetic site in this three-layer period structure has two nearest- and two next-nearest neighbor planes with ordered moments alternating in an up-down sequence. In an isotropic interaction model such an arrangement would yield zero net molecular field at the central paramagnetic site, and corresponding eigenstates which would be free-ion-like, i.e., sixfold degenerate. However, for the hybridization-mediated anisotropic interaction model in Eq. (1), the central paramagnetic site experiences a net molecular field (see Fig. 2 and related discussion in Ref. 3), yielding central site eigenstates which are three Kramers doublets, each consisting of a mixture of equal parts of up and down moment states.

This result can be understood by considering those diagonal matrix elements, $\left\langle\mu\left|\mathscr{H}_{\mathrm{CS}}\right| \mu\right\rangle$, of the CS Hamiltonian in Eq. (1) which give the largest contribution. Thus the $\left\langle\frac{5}{3}\left|\mathscr{H}_{\mathrm{CS}}\right| \frac{5}{2}\right\rangle$ matrix element at the central paramagnetic site, due to a pair of $n$th nearest-neighbor equivalent ordered sites with saturated up and down moments $\left(\left\langle L_{5 / 25 / 2}\right\rangle=\left\langle L_{-5 / 2-5 / 2}\right\rangle=1\right)$, respectively, is equal to $B_{5 / 25 / 2}^{5 / 25 / 2}(\theta)+B_{5 / 25 / 2}^{-5 / 2}-5 / 2(\theta)$. For general $\theta$ values this matrix element does not vanish because of the strong asymmetry of $B_{5 / 25}^{5 / 25 / 2}$ and $B_{5 / 25 / 2}^{-5 / 2}-5 / 2$ (see Fig. 1 of Ref. 3), which follows from the fact that the magnetic quantum numbers of the band and $f$ electrons are exchanged in the scattering involved in the hybridizationmediated mechanism. Moreover, due to the moment reversal symmetry of the interaction, one can easily show that the $\left\langle-\frac{5}{2}\left|\mathcal{H}_{\mathrm{CS}}\right|-\frac{5}{2}\right\rangle$ and $\left\langle\frac{5}{2}\left|\mathcal{H}_{\mathrm{CS}}\right| \frac{5}{2}\right\rangle$ matrix elements from a given pair of equivalent ordered sites are equal, and consequently the up and down (paramagnetic moment) states at the central paramagnetic site are doubly degenerate. All of the other matrix elements behave in similar fashion, and thus the paramagnetic site develops three Kramers doublets interacting through hybridization with the magnetically saturated states at the neighboring ordered sites. (As will be discussed in detail in Sec. IV, in the absence of CF interactions, or in the presence of weak $C F$ interactions with $B_{4}>0$, the ground-state doublet of the paramagnetic sites is predominantly of $\Gamma_{8}$ character. Since experimentally the $\mathrm{CF}$ ground state in the paramagnetic phase is ${ }^{19,21} \Gamma_{7}$, this leads us to anticipate that the hybridization-mediated two-ion interaction drastically modifies the states at the paramagnetic sites in the magnetically ordered structures compared to the cubic-crystal-field-split states above the ordering temperature.) The formation of doublets at the paramagnetic site leads to a ground-state energy below the free-ion energy $(E=0)$, so that it is advantageous for the paramagnetic site to have Kramers doublet states at low temperatures; as the temperature increases, the free energy of the paramagnetic site decreases less rapidly than that of the free-ion states, which have a clear entropy advantage, eventually crossing the free-ion energy at the Néel temperature. Furthermore, in order to compare the relative free energies of the pertinent magnetic structures without paramagnetic planes compared to that of the AFP 1 structure, we also have to consider the local magnetic environment seen by the magnetically ordered sites in the AFP 1 phase. An ordered site in the AFP 1 structure sees a sequence of nearest- and next-nearest neighbor planes consisting of alternating paramagnetic planes and ordered planes with reversed moments. Thus, because $\left\langle L_{5 / 25 / 2}\right\rangle=\left\langle L_{-5 / 2-5 / 2}\right\rangle \simeq \frac{1}{2}$ at a paramagnetic site, an ordered central site sees an effective number of $\frac{1}{2}$ and $\frac{3}{2}$ nearest- and next-nearest neighbor planes with parallel and antiparallel moments, respectively.

We now consider the relative free energies of the various pertinent magnetic structures at zero temperature. The way the zero-temperature free energy of an ordered site varies with the hybridization-mediated two-ion interactions $E_{1}, E_{2}$, and $E_{3}$ for the different magnetically ordered structures, can be determined by evaluating the contribution of the most dominant matrix elements to the MF energy. In the case of the two-ion Hamiltonian in Eq. (1) it has been shown ${ }^{3,13}$ that the most dominant contribution to the MF energy arises from the diagonal matrix elements corresponding to saturated moments. Thus the $T=0 \mathrm{MF}$ energy of an ordered site with an up moment in the AFP1, AF-I, AF-IA, and ferromagnetic (FM) structures, respectively, is given approximately by

$$
\begin{aligned}
& E_{\mathrm{AFP} 1}(\uparrow) \simeq-\left\{E_{1}\left[4 B_{5 / 25 / 2}^{5 / 25 / 2}(\pi / 2)+\frac{3}{2} B_{5 / 25 / 2}^{-5 / 2-5 / 2}(\pi / 4)+\frac{1}{2} B_{5 / 25 / 2}^{5 / 25 / 2}(\pi / 4)\right]\right. \\
& +E_{2}\left[4 B_{5 / 25 / 2}^{5 / 25 / 2}(\pi / 2)+\frac{3}{2} B_{5 / 25 / 2}^{-5 / 2-5 / 2}(0)+\frac{1}{2} B_{5 / 25 / 2}^{5 / 25 / 2}(0)\right] \\
& \left.+E_{3}\left[12 B_{5 / 25 / 2}^{-5 / 2-5 / 2}\left(\theta_{1}\right)+4 B_{5 / 2}^{5 / 25 / 2}\left(\theta_{1}\right)+6 B_{5 / 25 / 2}^{-5 / 2-5 / 2}\left(\theta_{2}\right)+2 B_{5 / 25 / 2}^{5 / 25 / 2}\left(\theta_{2}\right)\right]\right\}, \\
& E_{\mathrm{AF}-\mathrm{I}}(\uparrow) \simeq-\left\{4 E_{1}\left[B_{5 / 25 / 2}^{5 / 25 / 2}(\pi / 2)+2 B_{5 / 2}^{-5 / 2} 5 / 25 / 2(\pi / 4)\right]+2 E_{2}\left[2 B_{5 / 25 / 2}^{5 / 25 / 2}(\pi / 2)+B_{5 / 25 / 2}^{5 / 25 / 2}(0)\right]\right. \\
& \left.+8 E_{3}\left[2 B_{5 / 25 / 2}^{-5 / 2-5 / 2}\left(\theta_{1}\right)+B_{5 / 25 / 2}^{5 / 25 / 2}\left(\theta_{2}\right)\right]\right\} \text {, }
\end{aligned}
$$




$$
\begin{aligned}
E_{\mathrm{AF}-\mathrm{IA}}(\uparrow) \simeq- & \left\{4 E_{1}\left[B_{5 / 25 / 2}^{5 / 25 / 2}(\pi / 2)+B_{5 / 25 / 2}^{5 / 25 / 2}(\pi / 4)+B_{5 / 25 / 2}^{-5 / 2}-5 / 2(\pi / 4)\right]\right. \\
+ & 2 E_{2}\left[2 B_{5 / 25 / 2}^{5 / 25 / 2}(\pi / 2)+B_{5 / 2}^{-5 / 2-5 / 2}(0)\right] \\
& \left.+8 E_{3}\left[B_{5 / 25 / 2}^{5 / 25 / 2}\left(\theta_{1}\right)+B_{5 / 25 / 2}^{-5 / 2}-5 / 2\left(\theta_{1}\right)+B_{5 / 2}^{-5 / 2-5 / 2}\left(\theta_{2}\right)\right]\right\}, \\
E_{\mathrm{FM}}(\uparrow) \simeq-\{ & 4 E_{1}\left[B_{5 / 25 / 2}^{5 / 25 / 2}(\pi / 2)+2 B_{5 / 25 / 2}^{5 / 25 / 2}(\pi / 4)\right]+2 E_{2}\left[2 B_{5 / 25 / 2}^{5 / 25 / 2}(\pi / 2)+B_{5 / 2}^{5 / 25 / 2}(0)\right] \\
+ & \left.8 E_{3}\left[2 B_{5 / 25 / 2}^{5 / 25 / 2}\left(\theta_{1}\right)+B_{5 / 25 / 2}^{5 / 25 / 2}\left(\theta_{2}\right)\right]\right\},
\end{aligned}
$$

where $\theta_{1}=\cos ^{-1}(1 / \sqrt{6})$ and $\theta_{2}=\cos ^{-1}(2 / \sqrt{6})$.

Upon substituting the values of $B_{5 / 25 / 2}^{5 / 25 / 2}(\theta)$ and $B_{5 / 25 / 2}^{-5 / 2}-5 / 2(\theta)$ (see Fig. 2 of Ref. 3) in Eqs. (6)-(9), one obtains the expressions

$$
\begin{aligned}
& E_{\mathrm{AF} P 1}(\uparrow) \simeq-\left(0.97 E_{1}+1.06 E_{2}-0.40 E_{3}\right), \\
& E_{\mathrm{AF}-\mathrm{I}}(\uparrow) \simeq-\left(0.98 E_{1}+1.06 E_{2}-1.13 E_{3}\right), \\
& E_{\mathrm{AF}-\mathrm{IA}}(\uparrow) \simeq-\left(1.08 E_{1}+1.06 E_{2}+0.34 E_{3}\right), \\
& E_{\mathrm{FM}}(\uparrow) \simeq-\left(1.17 E_{1}+1.06 E_{2}+1.89 E_{3}\right) .
\end{aligned}
$$

Similarly, the zero-temperature MF energy of the paramagnetic site in the AFP1 is given approximately by the equation

$$
\begin{aligned}
& E_{\mathrm{AFP} 1}(\mathrm{para}) \simeq-\left\{2 E_{1}\left[B_{5 / 25 / 2}^{5 / 25 / 2}(\pi / 2)+B_{5 / 2}^{-5 / 2-5 / 2}(\pi / 2)+2 B_{5 / 25 / 2}^{5 / 25 / 2}(\pi / 4)+2 B_{5 / 25 / 2}^{-5 / 2-5 / 2}(\pi / 4)\right]\right. \\
& +E_{2}\left[B_{5 / 25 / 2}^{5 / 25 / 2}(\pi / 2)+2 B_{5 / 25 / 2}^{-5 / 2-5 / 2}(\pi / 2)+B_{5 / 25 / 2}^{5 / 25 / 2}(0)+B_{5 / 25 / 2}^{-5 / 2-5 / 2}(0)\right] \\
& \left.+4 E_{3}\left[2 B_{5 / 25 / 2}^{5 / 25 / 2}\left(\theta_{1}\right)+2 B_{5 / 25 / 2}^{-5 / 2}-5 / 2\left(\theta_{1}\right)+B_{5 / 25 / 2}^{5 / 25 / 2}\left(\theta_{2}\right)+B B_{5 / 25 / 2}^{-5 / 2-5 / 2}\left(\theta_{2}\right)\right]\right\} \text {, }
\end{aligned}
$$

which upon substituting the values of the $B$ 's assumes the simple form

$E_{\mathrm{AFP} 1}($ para $) \simeq-\left(0.29 E_{1}+0.28 E_{2}+0.36 E_{3}\right)$.

Equations (10)-(15) show that the zero-temperature free energy of the ferromagnetic and AF-IA structures increases linearly with decreasing (i.e., more negative) $E_{3}$, whereas that of the AF-I structure decreases linearly as $E_{3}$ decreases. On the other hand, the free energy of the ordered site in the AFP1 structure decreases less rapidly (by a factor of about 2.8) than that of the AF-I structure. Moreover, it is important to note from Eq. (15) that the zero-temperature free energy of the paramagnetic site in the AFP 1 phase decreases rather slowly with decreasing $E_{3}$, and is higher by a factor of about 3.5 than that of the ordered site in AFP 1 for a wide range of $E_{2} / E_{1}$ values $\left(0<E_{2}<E_{1}\right)$. Thus the zero-temperature free energy of the AFP 1 structure,

$$
E_{\mathrm{AFP} 1}=\frac{2}{3} E_{\mathrm{AF} P 1}(\uparrow)+\frac{1}{3} E_{\mathrm{AF} P 1}(\text { para }),
$$

decreases with decreasing $E_{3}$, but at a slower rate than that of the AF-I structure; and consequently the AFP1 structure can never become the ground state of the system at zero temperature. For the case of $E_{2}=E_{1}$, for example, the ground state is ferromagnetic for $\left|E_{3}\right|<0.057 E_{1}$, AF-I for $\left|E_{3}\right|>0.06 E_{1}$, and in the narrow intermediate range, $0.057<\left|E_{3} / E_{1}\right|<0.06$, the free energies of the ferromagnetic, AF-IA, and AF-I structures cross.

This large variation (both in sign and magnitude) of the energy of the ordered or paramagnetic site with $E_{3}$ in different magnetic structures, is a direct consequence of the highly anisotropic behavior of the matrix elements of the $B_{5 / 25 / 2}^{5 / 25}(\theta)$ and $B_{5 / 25 / 2}^{-5 / 2}-5 / 2(\theta)$ (which peak ${ }^{3}$ in the angular region near $\theta=\pi / 2$ ) in combination with the geometry of each magnetically odered structure. Thus, for an ordered site with an up moment, upon decreasing $E_{3}$, the AFP1 structure, which has effectively $\frac{3}{2}$ nearestneighbor (NN) and next nearest-neighbor (NNN) planes down and $\frac{1}{2}$ NN and NNN planes up, is less energetically favorable than the AF-I structure, which has 2 NN planes down and 2 NNN planes up; but it is more favorable than the AF-IA structure which has $1 \mathrm{NN}$ plane up and $1 \mathrm{NN}$ plane down and $2 \mathrm{NNN}$ planes down.

It should also be pointed out that because each of the four paramagnetic second nearest-neighbor sites to the central paramagnetic site within the para plane in the AFP 1 arrangement has doubly degenerate up and down paramagnetic moment states, and because of the large cancellation between the $B_{5 / 25 / 2}^{5 / 25 / 2}$ and $B_{5 / 25 / 2}^{-5 / 2}-5 / 2$ matrix elements at $\theta=\pi / 2$, the coefficient of the term proportional to $E_{2}$ in Eq. (14) is small, resulting in a variation of the ground-state energy of the paramagnetic site with $E_{2}$ which is slower by a factor of about 4 than of the ordered site. Thus, in contrast to the case ${ }^{3,13}$ of $\mathrm{CeBi}$ where the ratio $E_{2} / E_{1}$ was taken equal to unity in order to favor magnetic structures containing no paramagnetic planes, one must, in the case of $\mathrm{CeSb}$, choose a smaller $E_{2} / E_{1}(0.4-0.5)$ value in order to reach an energy compromise between the ordered and paramagnetic sites. This reduction of the $E_{2} / E_{1}$ value leads in turn to a lowering of the zero-temperature moment by an amount of about $10 \%$ compared to the free-ion value.

Above we have presented numerical results and physical arguments for the effect of the hybridization-mediated 
two-ion interactions on the ordered sites and on the formation of Kramers doublets at the paramagnetic sites at zero temperature in the absence of any crystal-field interactions. At nonzero temperatures, the paramagnetic sites, due to the formation of Kramers doublets, have a clear entropy advantage over the ordered sites, and hence one might have expected the AFP 1 structure to have the lowest free energy at high enough temperatures. However, it turns out (for the same reasons as presented above), that while the magnetic ordering temperatures of both the FM and AF-IA structures decrease linearly as the antiferromagnetic exchange interaction $E_{3}$ decreases, the Néel temperatures of both the AFP1 and the AF-I structures increase linearly with decreasing $E_{3}$. Furthermore, since only $\frac{2}{3}$ of the sites are ordered in the $\uparrow \downarrow P$ structure, the rate of increase of $T_{N}(\uparrow \downarrow P)$ is $\frac{2}{3}$ of that of $T_{N}(\uparrow \downarrow)$. Consequently, the AFP1 structure becomes disordered before it has the chance to cross the AF-I structure. Thus, in the absence of crystal-field interactions the free energy of the AFP1 is always higher than those of the other magnetically ordered structures considered, which contain no paramagnetic site.

\section{B. Crystal-field effects on paramagnetic and ordered sites and resulting stabilization \\ of magnetic structures containing paramagnetic planes}

We next investigate the role of the CF in helping to stabilize various magnetic structures containing paramagnetic layers in the high temperature part of the magnetically ordered regime. In particular, out of the six low-field AFP structures experimentally observed, $2,8,9,19$ we have considered for simplicity those two with the smallest periods, namely the AFP $3(\uparrow \uparrow \downarrow P \uparrow \downarrow \downarrow)$ and the AFP $1(\uparrow \downarrow P)$ structures ( in the notation of Refs. 2 and 19), which are observed ${ }^{8,19}$ in the temperature range of 12.2-14.8 $\mathrm{K}$ and $15.4-16 \mathrm{~K}$, respectively for $\mathrm{CeSb}$, i.e., the temperature range immediately below $T_{N}$.

The matrix elements of the cubic CF interaction of Eq. (2) in the free-ion basis-states representation are $\left\langle \pm \frac{5}{2}\left|H_{\mathrm{CF}}\right| \pm \frac{5}{2}\right\rangle=60 B_{4}, \quad\left\langle \pm \frac{3}{2}\left|H_{\mathrm{CF}}\right| \pm \frac{3}{2}\right\rangle=-180 B_{4}$, $\left\langle \pm \frac{1}{2}\left|H_{\mathrm{CF}}\right| \pm \frac{1}{2}\right\rangle=120 B_{4},\left\langle \pm \frac{5}{2}\left|H_{\mathrm{CF}}\right| \mp \frac{3}{2}\right\rangle=60 \sqrt{5} B_{4}$, and zero otherwise. The effect of the cubic CF interaction of a sign $\left(B_{4}>0\right)$ having the $\Gamma_{7}$ doublet below the $\Gamma_{8}$ quartet (favoring a $\langle 111\rangle$ easy axis ${ }^{5,36}$ ) is then to increase the diagonal $\left| \pm \frac{5}{2}\right\rangle$ and $\left| \pm \frac{1}{2}\right\rangle$ matrix elements, to decrease the diagonal $\left| \pm \frac{3}{2}\right\rangle$ matrix elements, and to introduce additional off-diagonal mixing between the $\left| \pm \frac{5}{2}\right\rangle$ and $\left|\mp \frac{3}{2}\right\rangle$ eigenstates of $J^{z}$, which in turn reduces both the ground-state moment $\left\langle J^{z}\right\rangle$ and the quadrupolar moment $\left\langle O_{2}^{0}\right\rangle$. Upon increasing the ratio $\Delta_{\mathrm{CF}} / E_{1}=360 B_{4} / E_{1}$, the zero-temperature expectation values (with respect to the molecular field eigenstates) of $\left\langle L_{ \pm 5 / 2 \pm 5 / 2}\right\rangle$ and $\left\langle L_{ \pm 5 / 2 \mp 3 / 2}\right\rangle$ decrease, that of $\left\langle L_{ \pm 3 / 2 \pm 3 / 2}\right\rangle$ increases, and $\left\langle L_{ \pm 1 / 2 \pm 1 / 2}\right\rangle$ is always vanishingly small. Consequently, the $\left\langle \pm \frac{5}{2} \pm \frac{5}{2}\right\rangle,\left\langle \pm \frac{1}{2} \mid \pm \frac{1}{2}\right\rangle$, and $\left\langle \pm \frac{5}{2} \mid \mp \frac{3}{2}\right\rangle$ matrix elements of the MF Hamiltonian increase, whereas the $\left\langle \pm \frac{3}{2} \mid \pm \frac{3}{2}\right\rangle$ matrix element of the MF Hamiltonian decreases as the magnitude of the crystal field increases.
The rate of change of the expectation values of the $\left\langle L_{\mu \nu}\right\rangle$ and of the molecular field Hamiltonian matrix elements with increasing crystal field, depends on whether the site is ordered or paramagnetic and on the type of the magnetically ordered structure. The net effect on the paramagnetic site of turning on the "bare" CF interaction, is to lower its ground-state energy from the value it has in the presence of only hybridization-mediated twoion interactions by an amount of approximately $-240 B_{4}=-2 \Delta_{\mathrm{CF}} / 3$ (this is the "bare" CF energy of a $\Gamma_{7}$ ground state compared to the free-ion energy), i.e.,

$$
E_{\mathrm{AF} P 1}\left(\text { para }, \Delta_{\mathrm{CF}}\right) \simeq E_{\mathrm{AFP} 1}\left(\text { para }, \Delta_{\mathrm{CF}}=0\right)-2 \Delta_{\mathrm{CF}} / 3,
$$

where $E_{\mathrm{AFP} 1}$ (para, $\Delta_{\mathrm{CF}}=0$ ) is given by Eq. (15). This expression is exact in the high $\Delta_{\mathrm{CF}} / E_{1}$ region $\left(\Delta_{\mathrm{CF}}>E_{1}\right)$ due to the predominance of the $C F$ interactions. In the low $\Delta_{\mathrm{CF}} / E_{1}$ region $\left(\Delta_{\mathrm{CF}}<E_{1}\right)$, as will be discussed in Sec. IV, the dominant hybridization-mediated two-ion interactions dress the ground-state Kramers doublet at the paramagnetic site so that it becomes $\Gamma_{8}$-like, and thus the energy of the paramagnetic site decreases with $\Delta_{C F}$ by a factor of about 1.5 less rapidly than that in Eq. (16). On the other hand, for the ordered site the effect of turning on the CF interaction is to raise its ground-state energy in the low $\Delta_{\mathrm{CF}} / E_{1}$ region $\left(\Delta_{\mathrm{CF}}<E_{1}\right)$, due to the increase of the $\left\langle \pm \frac{5}{2} \mid \pm \frac{5}{2}\right\rangle$ molecular field Hamiltonian matrix element, which yields the most dominant contribution to the MF energy. However, in the high $\Delta_{\mathrm{CF}} / E_{1}$ region $\left(\Delta_{\mathrm{CF}}>E_{1}\right)$, the $\left\langle \pm \frac{3}{2} \mid \pm \frac{3}{2}\right\rangle$ matrix elements are comparable in size with the $\left\langle \pm \frac{5}{2} \mid \pm \frac{5}{2}\right\rangle$ matrix elements, decreasing more rapidly with $\Delta_{\mathrm{CF}}$ than $\left\langle \pm \frac{5}{2} \mid \pm \frac{5}{2}\right\rangle$ increases. The net effect is the lowering of the MF energy of the ordered site with $\Delta_{\mathrm{CF}}$ for $\Delta_{\mathrm{CF}}>E_{1}$, but at a rate of about half that of the paramagnetic site. Thus, the presence of the CF interaction tends to favor predominantly the paramagnetic sites. Moreover, as will be discussed in Sec. IV, increasing the "bare" CF interaction compared to the hybridization-mediated two-ion interaction decreases the relative spacing between the two excited Kramers doublets (approaching the $\Gamma_{8}$ quartet behavior for large enough CF interaction), and thus enhances the entropy advantage of the paramagnetic sites in the hightemperature region. These two complementary effects, both of which arise from the presence of strong $C F$ interactions, are responsible for the stabilization of the AFP structures at high temperatures.

The effect of including the cubic CF interaction is shown in Fig. 1, where the Néel temperature, $T_{N}$, of the AF-I $(\uparrow \downarrow)$ and AFP $1(\uparrow \downarrow P)$ structures, and the temperature, $T_{2}$, at which the free energies of the AFP3 (more stable at low $T$ ) and AFP 1 (more stable at high $T$ ) structures cross, are plotted as function of the "bare" CF splitting, $\Delta_{\mathrm{CF}}$, (in units of $E_{1}$ ) with $E_{2} / E_{1}=0.4$ and $E_{3} / E_{1}=-0.28$. The separation between the $T_{N}(\uparrow \downarrow P)$ and $T_{2}$ curves shows the range of temperatures for which $G(\uparrow \downarrow P)$ is less than $G(\uparrow \uparrow \downarrow P \uparrow \downarrow \downarrow)$ as one varies the CF splitting. Also shown in Fig. 1 is the difference in free energies between the AFP1 and AF-I structures, 


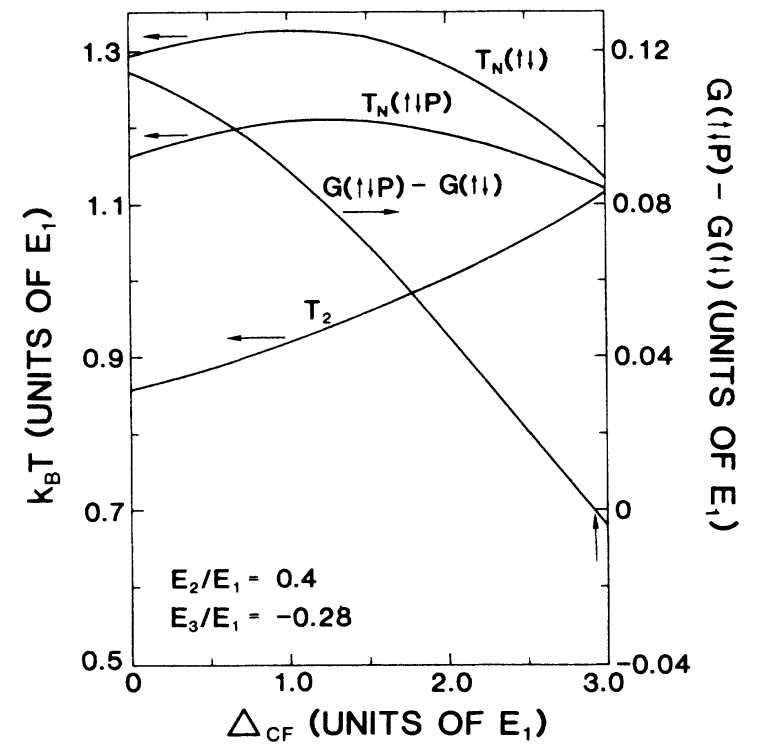

FIG. 1. Neel temperatures of the AF-I $(\uparrow \downarrow)$ and AFP1 $(\uparrow \downarrow P)$ structures, and transition temperature, $T_{2}$, from the AFP3 $(\uparrow \uparrow \downarrow P \uparrow \downarrow \downarrow)$ to the AFP 1 structure (with increasing temperature) in $\mathrm{Ce}^{3+}$ systems as a function of the "bare" cubic crystal-field $(C F)$ splitting ( $\Gamma_{7}$ ground state), with $E_{2}=0.4 E_{1}$, and $E_{3}=-0.28 E_{1}$. Also shown is the variation with crystalfield splitting of the difference in free energies between the AFP1 and AF-I structures, evaluated in the vicinity of $T_{N}$ $(\uparrow \downarrow P)\left[\right.$ at $\left.T-T_{N}(\uparrow \downarrow P)=-0.005 T_{N}(\uparrow \downarrow P)\right]$.

$G(\uparrow \downarrow P)-G(\uparrow \downarrow)$, evaluated in the vicinity of the Neel temperature of the AFP1 structure $\left[T-T_{N}(\uparrow \downarrow P)\right.$ $\left.=-0.005 T_{N}(\uparrow \downarrow P)\right]$, versus $\Delta_{\mathrm{CF}}$. [We choose the Néel temperature of the $\uparrow \downarrow P$ instead of the $\uparrow \downarrow$ structure in the evaluation of the $G$ 's because $T_{N}(\uparrow \downarrow)>T_{N}(\uparrow \downarrow P)$ in the whole $\Delta_{\mathrm{CF}}$ range in Fig. 1.] In the low $\Delta_{\mathrm{CF}} / E_{1}$ regime $\left(\Delta_{\mathrm{CF}}<E_{1}\right)$ the Néel temperature of the $\uparrow \downarrow$ structure is substantially higher than that of the $\uparrow \downarrow P$ structure (by an amount of about $0.1 E_{1}$, equivalent to about $14 \mathrm{~K}$ ), indicating that as the exchange interaction $E_{1}$ predominates it favors magnetic structures containing solely ordered planes. This result is further substantiated by the large positive value $\left(0.1 E_{1}\right)$ of $G(\uparrow \downarrow P)-G(\uparrow \downarrow)$ in the vicinity of $T_{N}(\uparrow \downarrow P)$, clearly showing that the AF-I structure is the stable structure in the whole temperature range below $T_{N}(\uparrow \downarrow)$. In the high $\Delta_{\mathrm{CF}} / E_{1}$ region $\left(\Delta_{\mathrm{CF}}>E_{1}\right)$ on the other hand, the difference both in Néel temperatures, $T_{N}(\uparrow \downarrow)-T_{N}(\uparrow \downarrow P)$, and in free energies, $G(\uparrow \downarrow P)-G(\uparrow \downarrow)$, decreases very rapidly with increasing $\Delta_{\mathrm{CF}} / E_{1}$, and for sufficiently large crystal-field strength the $\uparrow \downarrow P$ structure becomes the stable structure at high temperatures. The crossover from the AF-I to the AFP1 structure at $T-T_{N}(\uparrow \downarrow P)=0.005 T_{N}(\uparrow \downarrow P)$ occurs at $\Delta_{\mathrm{CF}}=1.92 E_{1}$ (indicated by a vertical arrow in Fig. 1) for $E_{2}=0.4 E_{1}$ and $E_{3}=-0.28 E_{1}$. As was discussed above, this crossover behavior is a consequence of the tendency of the $\mathrm{CF}$ interaction to favor predominantly the nonmagnetic planes. For $\Delta_{\mathrm{CF}}=3 E_{1}$, the system of $\mathrm{Ce}^{3+}\left(f^{1}\right)$ local moments undergoes three first-order phase transi- tions with increasing temperature from an AF-I to AFP3 to AFP1 structure, and finally becomes paramagnetic at the Néel temperature. (This is clearly illustrated in Fig. 2, which is discussed below.) However, when $\Delta_{\mathrm{CF}} \geq 3.02 E_{1}$, the temperature $T_{2}$ becomes higher than $T_{N}(\uparrow \downarrow P)$, the AFP 1 structure disorders before the AFP 3 structure has the chance to cross it, and consequently the system undergoes only two first-order phase transitions with increasing temperature from an AF-I to an AFP3 structure which becomes disordered at $T_{N}$. Thus, small perturbations on the delicate balance between hybridization mediated and CF interactions can lead to entirely different magnetic behavior.

The temperature variation of the free energy of the AF-I, AF-IA, AFP1, and AFP 3 structures is shown in Fig. 2 for $E_{2}=0.4 E_{1} E_{3}=-0.28 E_{1}$, and $\Delta_{C F}=3 E_{1}$. For comparison, also plotted in Fig. 2 is the temperature variation of the free energy of the paramagnetic $(P)$ and ordered $(M)$ planes in the AFP 1 structure. At low temperatures $\left(k_{B} T<0.8 E_{1}\right)$, even in the presence of strong $C F$ interactions, the free energy of the paramagnetic site is substantially higher than that of the ordered site due to the predominance of the exchange interactions which tend to favor mainly the ordered sites. Consequently, in the low-temperature region, the AF-I and AF-IA structures are more favorable than the AFP1 or AFP 3 structures, and the AFP 3 structure, containing a single para-

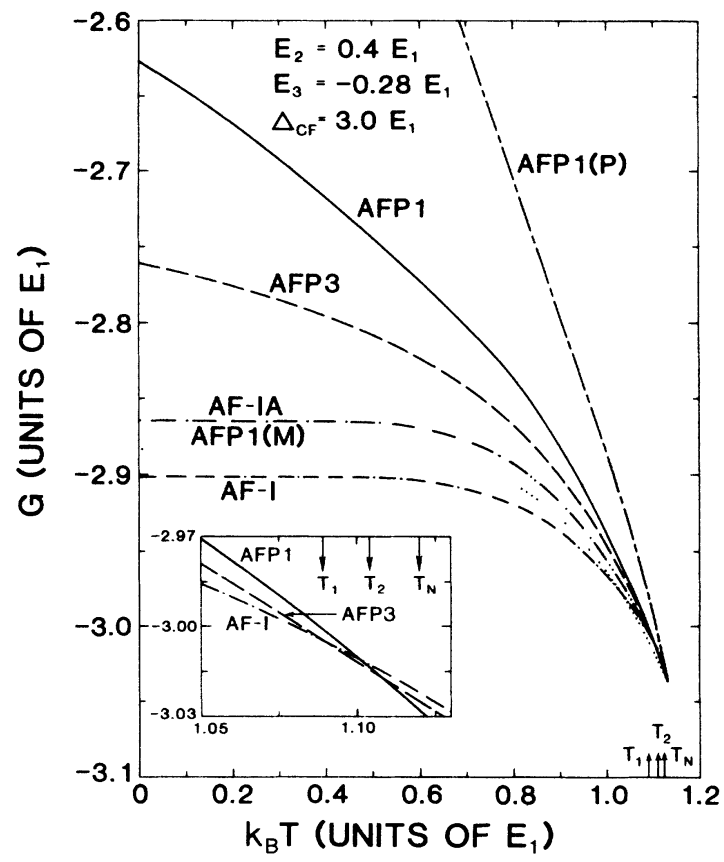

FIG. 2. Variation of free energy with temperature for different magnetic structures of $\mathrm{Ce}^{3+}$ fcc systems, with $E_{2}$ $=0.4 E_{1}, E_{3}=-0.28 E_{1}$, and $\Delta_{\mathrm{CF}}=3.0 E_{1}$. All structures are labeled as in Figs. 1 and 2 . Also shown for comparison is the temperature variation of the free energies of the paramagnetic $(P)$ and ordered $(M)$ sites for the AFP 1 structure. The model system undergoes first-order phase transitions with increasing temperature from the AF-I to the AFP3, to the AFP1 and finally to the paramagnetic phase, at the transition temperatures denoted by $T_{1}, T_{2}$, and $T_{N}$, respectively. 
layer every seven layers, is more favorable than the AFP1 structure which contains one para-layer every three layers. Moreover, owing to the significant antiferromagnetic exchange interaction, $E_{3}$, which tends to stabilize AF moment arrangements, the free energy of an up moment site in the AFP 1 structure (which effectively sees $\frac{3}{2} \mathrm{NN}$ planes with moment down and $\frac{1}{2} \mathrm{NN}$ plane up) is lower than that for an up site in the AF-IA structure (with one NN plane up and one NN plane down), and higher than that for an up site in the AF-I structure (with two NN planes down). With increasing temperature, the free energy of the AFP1 paramagnetic site decreases more rapidly than that of the ordered site because of the entropy advantage (three Kramers doublets); and thus, as one can clearly see in the inset of Fig. 2, the free energy of the AF-I structure, which is stable at low temperature, crosses the energy of the AFP3 structure at $T_{1}=1.089 E_{1}$, the latter energy crossing in turn that of the AFP1 structure at $T_{2}=1.104 E_{1}$, which becomes finally paramagnetic at $T_{N}=1.12 E_{1}$ with a first-order transition. Note that the $(\uparrow \uparrow \downarrow \downarrow)$ AF-IA structure, which is usually the experimentally observed ${ }^{2,8}$ low-temperature phase in $\mathrm{CeSb}$, has higher free energy relative to the $(\uparrow \downarrow \uparrow \downarrow)$ AF-I structure over the whole temperature range, becoming paramagnetic at $1.09 E_{1}$. (In this regard, it may be significant that for $\mathrm{CeBi}$, which also usually has an AF-IA structure at the lowest temperatures, the low temperature magnetic structure is sample dependent and can be ${ }^{8,10}$ AF-I.)

We could decrease $\left|E_{3}\right|$ (into the range -0.06 $<E_{3} / E_{1}<-0.05$ ) and $\Delta_{\mathrm{CF}}$ (into the range 0.5 $\left.<\Delta_{\mathrm{CF}} / E_{1}<0.85\right)$ relative to $E_{1}$, and obtain a groundstate AF-IA magnetic structure in agreement with experiment for $\mathrm{CeSb}$. (This sensitivity of magnetic structure to $E_{3}$ and $\Delta_{\mathrm{CF}}$, as discussed above, is associated with the highly anisotropic nature of the matrix elements giving the exchange interaction, in combination with the specific geometry of the various magnetic structures.) However, as shown in Sec. III A, this choice of range parameters and crystal-field strength would have decreased the ordering temperatures of both the AFP 1 and AFP3 structures so that the system would disorder before either of these became the stable magnetic structure. Thus, attempting to bring the low-temperature part of the phase diagram into better agreement with experiment, adversely affects the higher-temperature agreement with experiment. If one stabilizes an AF-IA structure at low temperatures, then with increasing temperature one has a first-order transition to an AF-I structure before disordering at $T_{N}$, i.e., behavior such as experimentally occurs in CeBi. This discrepancy with experiment for the ground-state magnetic structure of $\mathrm{CeSb}$ may arise from the omission of the cubic part of the hybridization potential (taken as spherically symmetric in obtaining the hybridization-mediated exchange) or of some weak interaction which has not been included in our calculations such as higher multipolar couplings between cerium ions brought about when the hybridization-induced interactions are treated to higher order in perturbation theory.

Our numerical results for the zero-temperature molecular-field energy levels (in units of $E_{1}$ ) of the AF-I, AF-IA, and AFP1 structures are shown in Fig. 3, for $E_{2}=0.4 E_{1}, E_{3}=-0.28 E_{1}$, and $\Delta_{\mathrm{CF}}=3 E_{1}$. For such a relatively large $\mathrm{CF}$ splitting, the ordered site ground-state energies in all three magnetic structures are almost identical $\left(-2.9 E_{1}\right)$ and much lower than the paramagnetic site energy of $-2.1 E_{1}$. Such a situation cannot occur in $\mathrm{CeBi}$, because the CF splitting $(\sim 8 \mathrm{~K})$ is much smaller ${ }^{20}$ than the Néel temperature $(\sim 25 \mathrm{~K})$ which directly scales with $E_{1}$. In particular, for the same $E_{2} / E_{1}$ and $E_{3} / E_{1}$ values and in the absence of a crystal field, the ordered site ground-state energies of the AF-I, AF-IA, and AFP1 structures are $-2.0 E_{1},-1.7 E_{1}$, and $-1.87 E_{1}$, respectively, compared to the rather high paramagnetic site ground-state energy of $-0.43 E_{1}$. This illustrates the statement made above, that upon increasing the CF strength $\left(\Gamma_{7}\right.$ ground state) relative to the hybridizationmediated exchange interactions, the paramagnetic site ground-state energy decreases more rapidly than that of the ordered site. In addition, because of the large CF splitting the zero-temperature moments, $\left\langle J^{z}\right\rangle$, of the AF-I, AF-IA, and AFP1 structures are greatly suppressed from the almost saturated value $\left(\frac{5}{2}\right)$, to the values of $1.04,0.28$, and 0.60 , respectively.

The large reduction of the $\mathrm{Ce}^{3+}$ local moment due to the CF effects can be overcome by introducing a weak single-ion term in the Hamiltonian which mixes free-ion states that have the same magnetic quantum numbers. Equilibrium lattice distortions associated with magnetic ordering provide a single-site coupling mechanism between the magnetic ions and the lattice strain and give such a single-site state mixing term. Even though one

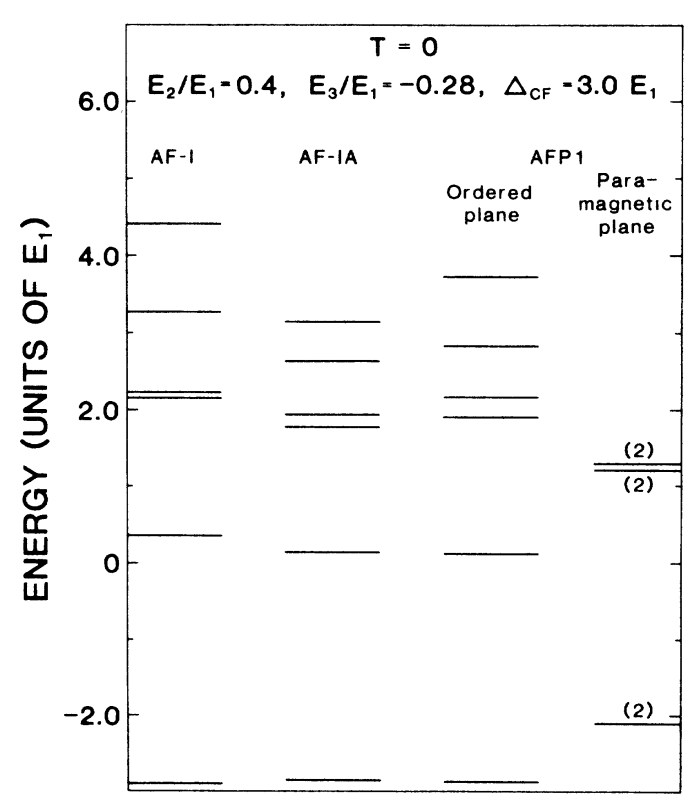

FIG. 3. Zero-temperature molecular-field energy level schemes for AF-I, AF-IA, and AFP1 magnetic structures of fcc $\mathrm{Ce}^{3+}$ systems, with $E_{2} / E_{1}=0.4, \quad E_{3} / E_{1}=-0.23$, and $\Delta_{\mathrm{CF}}=3.0 E_{1}$. (AF-IA denotes the $\uparrow \uparrow \downarrow \downarrow$ antiferromagnetic structure; AF-I and AFP1 structures are labeled as in Fig. 1.) 
could in principle consider the general case of nonuniform strain, in order to keep the number of parameters to a minimum, we consider for simplicity the case of a small uniform [001] tetragonal distortion $\delta=c / a-1$, which introduces in the Hamiltonian a magnetic ion-tetragonal lattice strain coupling term and a harmonic elastic energy term of the form ${ }^{37}$

$$
\mathscr{H}_{d}=x B_{4} \delta O_{2}^{0}+k_{0} \delta^{2} / 2
$$

where $O_{2}^{0}$ is a Stevens operator equivalent, ${ }^{35} x$ is a constant of the order of $10^{3}$, and $k_{0}$ is an effective elastic constant. (A small [001] tetragonal distortion has indeed been experimentally observed ${ }^{38}$ in the cerium monopnictides.) Minimization of the total energy with respect to $\delta$ yields the expression

$$
\mathscr{H}_{d}=-Q\left\langle O_{2}^{0}\right\rangle O_{2}^{0}+\frac{1}{2} Q\left\langle O_{2}^{0}\right\rangle^{2},
$$

where the quantity $Q=\left(x B_{4}\right)^{2} / k_{0}$, rather than $k_{0}$, can be treated as an independent phenomenological parameter. The distortion $\delta$ is related to $Q$ by $\delta=-\left(Q / x B_{4}\right)\left\langle O_{2}^{0}\right\rangle$. However, besides increasing the local moments, the distortional term tends also to lower the ordered site ground-state energy more rapidly than that of the paramagnetic site, and consequently one has to readjust the CF interaction. Thus, if $Q=0.01 E_{1}$, then $\Delta_{\mathrm{CF}}$ must be increased from $E_{1}$ to $1.1 E_{1}$ so as to reproduce a magnetic behavior similar to that in the absence of $Q$, but with a saturated zero-temperature moment. Using a value of $Q$ corresponding to the experimental distortion ${ }^{38}$ $\left(-1.2 \times 10^{-3}\right)$ increases the ordered moment to about $80 \%$ of the saturated value, considerably better agreement with experiment than the values found without the distortional term. The increase of moment slows down for further increase of distortion, so that to essentially reach moment saturation requires an increase of the distortion by about a factor of 10 . For simplicity and to keep the number of parameters to a minimum, the numerical results reported throughout the rest of this section are for the $Q=0$ case.

The variation of the average sublattice moment $\left\langle J^{z}\right\rangle$, normalized to its zero-temperature value with temperature, is shown in Fig. 4 for the AF-I, AFP3, and AFP1 structures, with $E_{2}=0.4 E_{1}, \quad E_{3}=-0.28 E_{1}$, and $\Delta_{\mathrm{CF}}=3 E_{1}$, along with the experimental ${ }^{19}$ neutron scattering results per ordered $\mathrm{Ce}^{3+}$ sites. For this parameter set our model fcc $\mathrm{Ce}^{3+}\left(f^{1}\right)$ system undergoes three successive first-order phase transitions from a type-AF-I $(0-16.7 \mathrm{~K})$ to a type-AFP3 $(16.7-(16.95 \mathrm{~K})$ to a typeAFP 1 (16.95-17.18 K) structure and finally to the paramagnetic phase. The value of $E_{1}=15.35 \mathrm{~K}$ is chosen to match the experimentally observed ${ }^{19}$ Néel temperature, $T_{N}=17.2 \mathrm{~K}$; while $\Delta_{\mathrm{CF}}$ is taken as $46.04 \mathrm{~K}$, compared to the experimental high-temperature (above $T_{N}$ ) CF splitting of $38 \pm 3 \mathrm{~K},{ }^{20}$ for CeSb. The Néel temperature of $17.2 \mathrm{~K}$ we used in plotting Fig. 4 was obtained from recent neutron scattering experiments (Fig. 4 of Ref. 19), and is slightly higher than the values of 16.2 $\mathrm{K}$ reported in Ref. 2 . The neeed to choose a theoretical $\Delta_{C F}$ value higher than the experimental one is due to the highly nonlinear dependence of the temperature variation

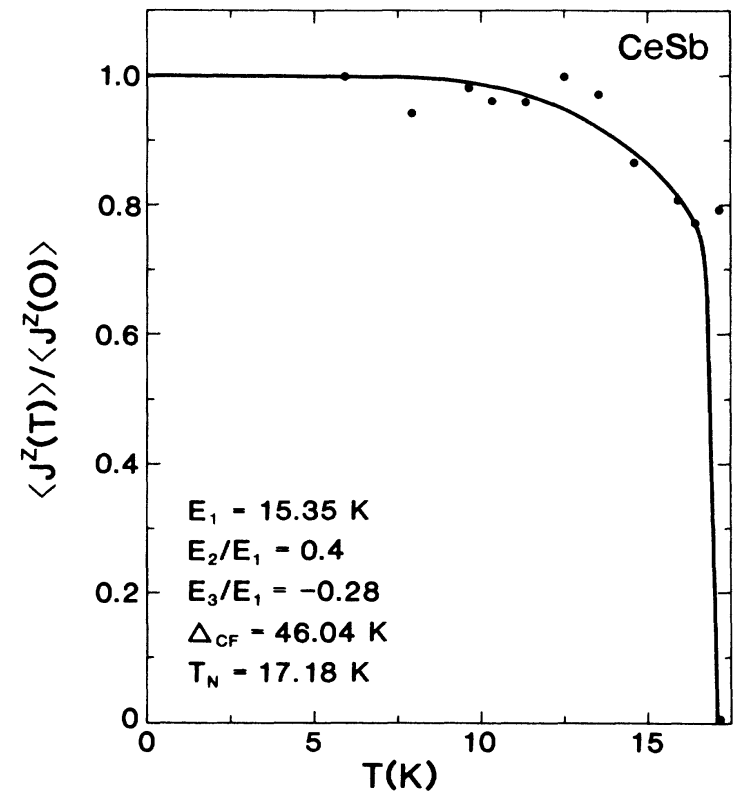

FIG. 4. Variation of the average sublattice magnetic moment of $\mathrm{CeSb}$ (normalized to its zero-temperature value) with temperature. The solid circles denote the results from neutronscattering experiments (Ref. 19); the theoretical curve has been obtained with $E_{2}=0.4 E_{1}, E_{3}=-0.28 E_{1}$, and $\Delta_{\mathrm{CF}}=3.0 E_{1}$. The fcc $\mathrm{Ce}^{3+}$ system undergoes first-order phase transitions from an AF-I $(0-16.7 \mathrm{~K})$ to an AFP3 $(16.7-16.95 \mathrm{~K})$ to an AFP 1 (16.95-17.18 K) phase. We have set $E_{1}=15.35 \mathrm{~K}$ to match the experimental value of $T_{N}=17.2 \mathrm{~K}$, which yields $\Delta_{\mathrm{CF}}=46.04 \mathrm{~K}$.

(especially in the vicinity of $T_{N}$ ) of the free energies and magnetization upon $\Delta_{C F}$, which does not allow one to match exactly both the $T_{N}$ and $\Delta_{\mathrm{CF}}$ values, and also to reproduce the main features of the phase diagram. The theoretical magnetization curve is in very good agreement with experiment at all temperatures, even though the experimental points in the temperature range of 8-15 $\mathrm{K}$ refer to various $A F P$-type structures and the theoretical ones for simplicity correspond to the AF-I structure. For the theoretical moment in the AFP3 $(\downarrow \downarrow \uparrow P \downarrow \uparrow \uparrow)$ structure we have plotted the average of the moments of the three ferromagnetically aligned planes. The moments on the three planes in the $\downarrow \downarrow \uparrow \downarrow \downarrow \uparrow \uparrow$ sequence are not identical, but are symmetrically arranged with respect to the central paramagnetic plane, i.e., the two inner planes which are nearest neighbor to the paramagnetic plane have the largest moment, with the moment decreasing monotonically as one goes to the outer planes. The ratio of the moment on one of the inner planes to that on the two outer planes is about 1.3 and 1.6, respectively, at zero temperature, but changes significantly as the temperature is raised. The AFP3 and AFP 1 structures have been experimentally observed ${ }^{8}$ in the temperature range of about $12.2-14.8 \mathrm{~K}$ and $15.4-16 \mathrm{~K}$, respectively. However, thermal moment fluctuations, which are entirely neglected in our simple molecular field treatment, have a substantial effect on the free energies within the critical region, lowering the temperatures at which the transitions occur. 
Although the magnetic moment $\left\langle J^{z}\right\rangle$ is zero at the paramagnetic sites in the AFP1 and AFP 3 structures, the thermal average of the quadrupole moment, $\left\langle O_{2}^{0}\right\rangle$, in the absence of CF interactions, is fairly large (compared to the saturated value of 10 ) at low temperatures and decreases rapidly with increasing temperature in contrast to the rather slow temperature variation of $\left\langle J^{z}\right\rangle$ at the ordered sites. Our numerical results for the variation of $\left\langle O_{2}^{0}\right\rangle$ with temperature in the presence of the crystal field $\left(\Delta_{\mathrm{CF}}=3 E_{1}\right)$ are displayed in Fig. 5 using the same interaction parameter ratios as those used in Figs. 2 and 3 $\left(E_{2} / E_{1}=0.4\right.$ and $\left.E_{3} / E_{1}=-0.28\right)$. It should be noted that we have plotted the average sublattice quadrupole moment (solid curve) for the AF-I, AFP3, and AFP1 structures, i.e., $\left\langle O_{2}^{0}(\uparrow \downarrow P)\right\rangle=\frac{2}{3}\left\langle O_{2}^{0}(\uparrow)\right\rangle+\frac{1}{3}\left\langle O_{2}^{0}(P)\right\rangle$ which are the stable structures in the temperature range of $0-1.089,1.089-1.104$, and 1.104-1.12 (in units of $\left.E_{1}\right)$, respectively. For comparison, also shown in Fig. 5 is the temperature variation of $\left\langle O_{2}^{0}\right\rangle$ associated with the paramagnetic $(P)$ and ordered $(\boldsymbol{M})$ planes (dashed curves) of the AFP $1(\uparrow \downarrow P)$ structure, respectively, in the whole temperature range, even though the $\uparrow \downarrow P$ structure is stable only in a very narrow temperature window (1.104-1.12, in units of $\left.E_{1}\right)$. As one can see from Fig. 5, the presence of the crystal field reduces the ground-state quadrupole moment (as in the case of $\left\langle J^{z}\right\rangle$ ) at both paramagnetic and ordered sites, and tends to decrease the temperature variation of $\left\langle O_{2}^{0}\right\rangle$ over a wide range of tem-

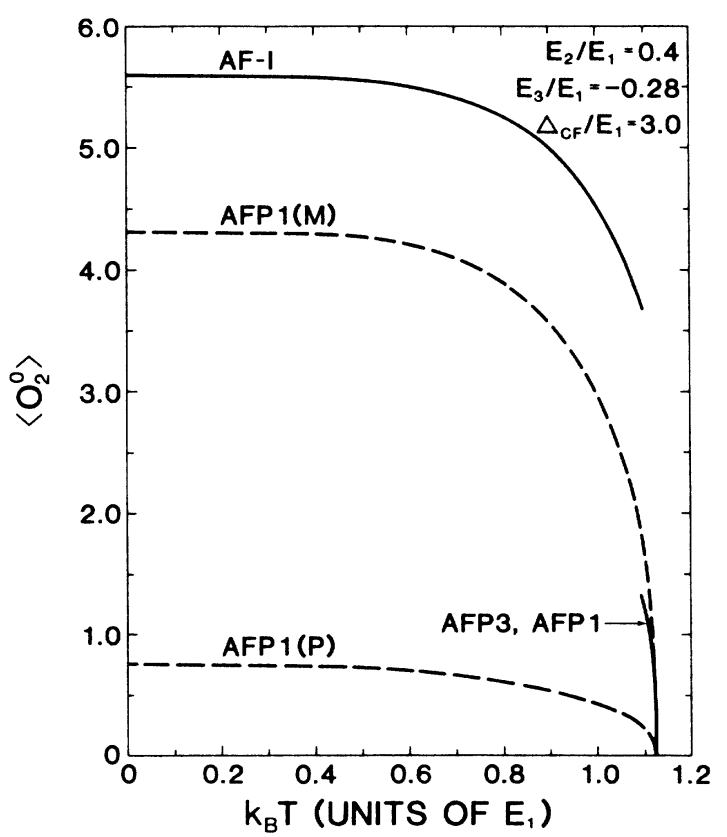

FIG. 5. Temperature variation of quadrupole moment for fcc $\mathrm{Ce}^{3+}$ systems, with $E_{2}=0.4 E_{1}, \quad E_{3}=-0.28 E_{1}, \quad$ and $\Delta_{\mathrm{CF}}=3.0 E_{1}$. The average sublattice quadrupole moment has been plotted for the AF-I, AFP3 and AFP1 phases over the temperature range within which these structures have the lowest free energy (solid curve). The structures are labeled as in Fig. 1. Also plotted for comparison (dashed curves) is the temperature variation of the ordered $(M)$ and paramagnetic $(P)$ site quadrupole moment for the AFP1 structure. peratures. Furthermore, the large suppression of $\left\langle\mathrm{O}_{2}^{0}\right\rangle$ at the paramagnetic sites in the AFP 1 structure $(\sim 0.75$ at low $T$ ) indicates that the paramagnetic layers would participate rather weakly in a lattice distortion in the presence of strong $\mathrm{CF}$ interactions.

\section{CRYSTAL-FIELD DRESSING}

The "bare" point-charge-like cubic crystal field in Eq. (2) is modified, or "dressed," by the hybridization between the quasilocalized ionic $f$ states and the band. This dressing arises through single-site ${ }^{31,39,40}$ and twoion $^{3,13}$ hybridization interactions. The single-site hybridization has been shown ${ }^{31,39,40}$ to be the predominant effect responsible for the large reduction of the cubic CF splitting in the paramagnetic phase in $\mathrm{CeBi}$ and $\mathrm{CeSb}$ from the values expected from the point-charge model. In particular, the single-site hybridization affects the $\Gamma_{8}$ state predominantly pushing it down in energy toward the $\Gamma_{7}$ state, thus reducing the CF splitting in the paramagnetic phase. Since in this paper we primarily concentrate on explaining magnetic ordering effects, we shall be concerned solely with the CF dressing arising from the anisotropic two-ion interaction.

The effect that varying the "bare" CF splitting $\Delta_{\mathrm{CF}}$ (with a "bare" $\Gamma_{7}$ ground state) has on the zerotemperature molecular-field energies and wave functions of the paralayers in the AFP1 structure is shown in Fig. 6 , with $E_{2} / E_{1}=0.4$ and $E_{3} / E_{1}=-0.28$. Upon increasing the ratio $\Delta_{\mathrm{CF}} / E_{1}$ the relative spacing between the two excited doublets decreases, with the doublets almost merging into a single $\Gamma_{8}$ quartet for large enough $\mathrm{CF}$

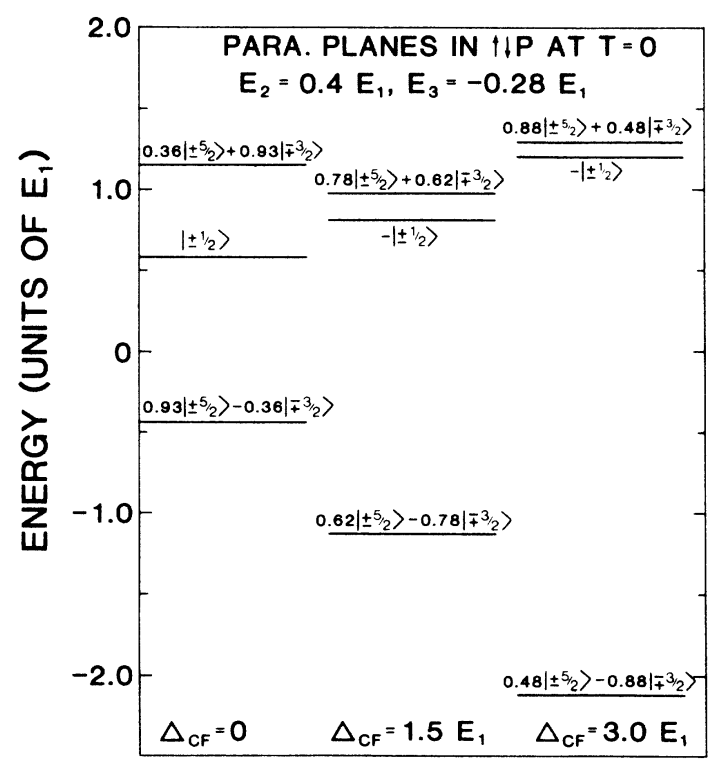

FIG. 6. Zero-temperature molecular-field (MF) energy levels and eigenstates (Kramers doublets) for the paramagnetic planes of the AFP1 $(\uparrow \downarrow P)$ structure in fcc $\mathrm{Ce}^{3+}$ systems, with $E_{2}=0.4 E_{1}, E_{3}=-028 E_{1}$, and varying values of the "bare" cubic crystal-field splitting ( $\Gamma_{7}$ ground state). The MF states are expressed in terms of the angular momentum eigenstates quantized along [001]. 
splitting. It should be pointed out that for weak $\mathrm{CF}$ interaction $\left(\Delta_{\mathrm{CF}}<E_{1}\right)$ the ground-state doublet is predominantly of $\Gamma_{8}$ type (a nearly $\left|\frac{5}{2}\right\rangle$ state), clearly indicating that the hybridization-mediated two-ion interaction, which favors a $\langle 001\rangle$ saturated moment alignment, has significantly "dressed" the ground state, dramatically altering its nature. (The "bare" $\mathrm{CF} \Gamma_{8}$ states are $0.91\left| \pm \frac{5}{2}\right\rangle+0.41\left|\mp \frac{3}{2}\right\rangle$ and $\left| \pm \frac{1}{2}\right\rangle$; the "bare" $\Gamma_{7}$ states are $0.41\left| \pm \frac{5}{2}\right\rangle-0.91\left|\mp \frac{3}{2}\right\rangle$.) The degree of dressing of the CF states of the paralayers depends, of course, on the relative strength of the $\mathrm{CF}$ and two-ion exchange interaction, decreasing as the ratio $\Delta_{\mathrm{CF}} / E_{1}$ increases.

As far as the position of the molecular field energy levels is concerned, as can be seen in Fig. 6 for a given $\Delta_{\mathrm{CF}} / E_{1}$ value, the two-ion coupling predominantly affects the two closely spaced excited doublets pushing them up in energy (by an amount of about 0.35 in units of $E_{1}$ ) from their "bare" $\mathrm{CF}$ value of $\Delta_{\mathrm{CF}} / 3$ (in units of $E_{1}$ ), whereas the ground-state energy is pushed slightly down (by an amount of about 0.1 in units of $E_{1}$ ) from its "bare" $C F$ value of $-2 \Delta_{C F} / 3$ (in units of $E_{1}$ ). It is also clear from Fig. 6 that the relative spacing between the two excited Kramers doublets decreases as the "bare" CF interaction increases (eventually almost merging into a single $\Gamma_{8}$ quartet for large enough $C F$ strength), thus enhancing the entropy advantage of the paramagnetic sites in the high-temperature regime.

The dressed CF splittings, $\Delta_{1}^{*}$ and $\Delta_{2}^{*}$, respectively, between the ground state and the two excited doublets of the paraplanes in both the AFP1 and AFP3 structures are found to be temperature independent below the ordering temperature, $T_{N}$, within an accuracy of about $1 \%$, in agreement with experiment. ${ }^{20}$ Thus the net effect of the two-ion dressing is an enhancement of the CF splitting in the ordered state relative to its high-temperature "bare" value in the paramagnetic phase. This enhancement of the CF splitting in the paraplanes has been observed in inelastic neutron-scattering experiments, ${ }^{19,20}$ revealing a CF splitting of $43 \pm 2 \mathrm{~K}$ at $T=14 \mathrm{~K}$ relative to the high-temperature value of $37 \pm 2 \mathrm{~K}$ at $T=20 \mathrm{~K}$ and hence an enhancement factor of about 1.16. Using the same set of parameter values as that used in Fig. 2, and matching the experimental ${ }^{8} T_{N}=16.2 \mathrm{~K}$, which yields $E_{1}=14.4 \mathrm{~K}$ and a bare $\Delta_{\mathrm{CF}}=43.2 \mathrm{~K}$, we find $\Delta_{1}^{*}=48.2$ $\mathrm{K}$ and $\Delta_{2}^{*}=48.8 \mathrm{~K}$ at $T=14 \mathrm{~K}$, and thus an enhancement factor of about 1.12 in good agreement with experiment. Even though our calculations yield a double-peak structure, the splitting is small $(\sim 0.6 \mathrm{~K})$, so it is not surprising that experimentally one observes a single broad inelastic peak.

\section{FULLY NONMAGNETIC GROUND STATE}

The results of our investigation into the role that the crystal field plays in helping to stabilize the coexistence of paramagnetic and magnetically ordered sites in $\mathrm{CeSb}$ in the high-temperature part of the magnetically ordered region, motivated us to address the following question: Can the presence of sufficiently strong crystal-field interaction relative to a nonvanishing hybridizationmediated two-ion interaction give rise to a new type of ground state which is fully paramagnetic, i.e., the ground state at every site is Kramers doublet? [Note that this is not a Kondo-like (singlet) state, i.e., not a state resulting from the quenching of the local $f$ moment by the spin polarization of those conduction electrons which, through interacting with moderately delocalized $4 f$ electrons, form Kondo resonance states at the Fermi level.] Thus we consider the case in which the Kondo temperature, $T_{K}$, associated with the $f^{1} \mathrm{Ce}^{3+}$ ion is substantially smaller than the temperature, $T_{\mathrm{CS}}$, associated with the hybridization-mediated exchange interaction $\left(T_{\mathrm{CS}} \sim E_{1}\right)$, and consequently upon lowering the temperature the state with nonzero ionic moment precedes the formation of the singlet Kondo ground state. Since the rate of decrease of the ground-state energy of the ordered site with $B_{4}$ is lower by a factor of about 1.6 relative to that of the paramagnetic site for $E_{1}<\Delta_{\mathrm{CF}} \leq 3 E_{1}$, we felt that it is conceivable that for a still larger $\Delta_{\mathrm{CF}} / E_{1}$, the groundstate energy of the paramagnetic site would eventually cross that of the ordered site, provided that both variation rates did not change appreciably. However, as it turns out, the rate of decrease of the ground-state energy of the ordered site with $B_{4}$ increases and becomes equal to that of the paramagnetic site $\left(d E / d B_{4}=-240\right)$ for $\Delta_{\mathrm{CF}}>3 E_{1}$, and thus for nonzero two-ion interaction the ordered site remains always more favorable than the paramagnetic site at zero temperature.

In the process of investigating the possibility of a totally paramagnetic ground state we encountered a new and interesting physical situation. As pointed out earlier in Sec. III B, upon increasing $\Delta_{\mathrm{CF}} / E_{1}$ (with $B_{4}>0$ ), the expectation value $\left\langle L_{ \pm 5 / 2 \pm 5 / 2}\right\rangle$ decreases, $\left\langle L_{ \pm 3 / 2 \pm 3 / 2}\right\rangle$ increases, while $\left\langle L_{ \pm 1 / 2 \pm 1 / 2}\right\rangle$ is vanishingly small. Consequently, at zero temperature the ordered moment for a magnetic structure longitudinally polarized along [001] decreases; whereas the ordered moment (transverse to the modulation direction) for a transversely polarized structure increases due to the off-diagonal mixing. This can be seen, for example, by considering a longitudinally polarized moment pointing up a [001] modulation direction

$$
\left\langle J^{z}(\uparrow)\right\rangle=\frac{5}{2}\left\langle L_{5 / 25 / 2}\right\rangle-\frac{3}{2}\left\langle L_{-3 / 2-3 / 2}\right\rangle .
$$

This decrease of moment for the longitudinal structures is illustrated in Fig. 7 where the zero-temperature [001] component of the moment is plotted as function of $\Delta_{\mathrm{CF}} / E_{1}$ (with $\Gamma_{7}$ ground state) for the type-I $(\uparrow \downarrow)$ and type-IA $(\uparrow \uparrow \downarrow \downarrow)$ longitudinally polarized structures with $E_{1}=E_{2}=\left|E_{1}\right|$ and $E_{3}=-0.3 E_{1}$. Also shown in the same figure is the variation of the corresponding groundstate energy of the type-I and type-IA structures with $\Delta_{\mathrm{CF}} / E_{1}$. Note that the rate of decrease of $\left\langle J^{z}\right\rangle$ with $\Delta_{\mathrm{CF}} / E_{1}$ for the AF-IA magnetic structure is much more rapid than that for the AF-I structure. Thus even though the AF-I structure is the ground state for $\Delta_{C F}<3.12 E_{1}$ with a rather large value of the zero-temperature moment; when the AF-IA structure becomes the ground state for $\Delta_{\mathrm{CF}} \geq 3.12 E_{1}$, the moment is extremely small. Most interestingly, we find that for a value of $\Delta_{C F}$ slightly above the point (indicated by an arrow in Fig. 7) at which the AF-IA becomes the ground state, its [001] moment 


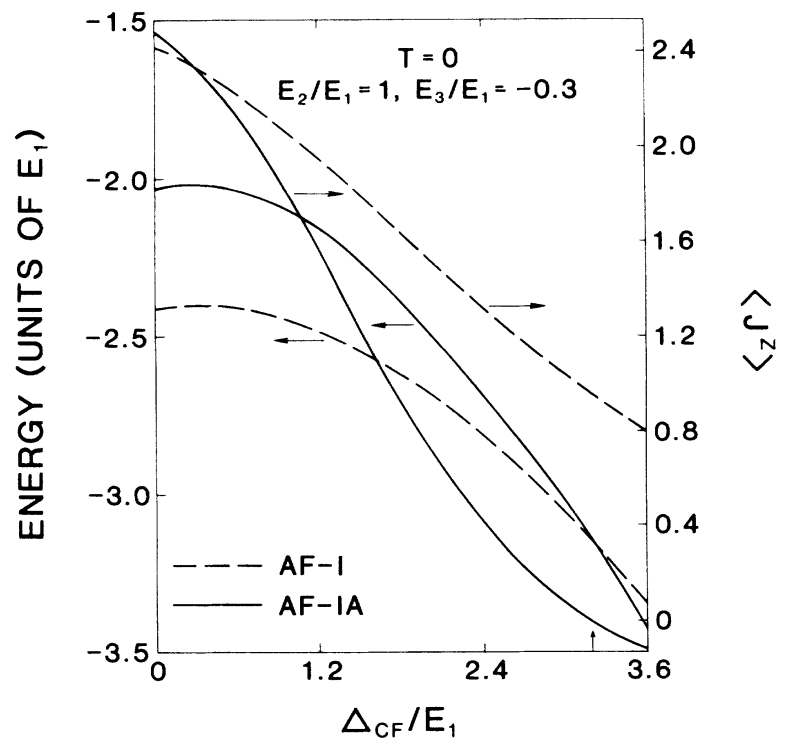

FIG. 7. Ground-state energy for the type-I and type-IA longitudinally polarized structures (as labeled in Fig. 1) as function of the crystal-field splitting (with $\Gamma_{7}$ ground state) relative to the nearest-neighbor hybridization-mediated two-ion interaction, with $E_{2}=E_{1}$ and $E_{3}=-0.3 E_{1}$. Also shown is the variation with $\Delta_{\mathrm{CF}} / E_{1}$ of the zero-temperature longitudinal component of the moment along the [001] direction of modulation for these structures.

vanishes, yielding a totally nonmagnetic singlet ground state, which, however, is not Kondo-like. (For a very narrow range of $\Delta_{\mathrm{CF}} / E_{1}$ values about this point the [001] moment is vanishingly small.) Moreover, in the presence of such a large $\Delta_{\mathrm{CF}}$ value, we find that the transversely polarized [110] AF-IA structure (with moments along the face diagonal transverse to the modulation vector along the cube edge) with the rather large moment components $\left(\left\langle J^{x}\right\rangle=\left\langle J^{y}\right\rangle \simeq 0.77\right)$ has the same free energy as the longitudinally polarized structure over a wide temperature range, i.e., for $k_{B} T<0.4 E_{1}$ (where the Néel temperature is of the order of $\left.E_{1}\right)$. Thus even though at high temperatures $\left(k_{B} T>0.4 E_{1}\right)$ the high-moment transversely polarized state has the lowest free energy, one encounters a peculiar lower-temperature physical phenomenon where the system fluctuates between two differently polarized states of equal free energies but dramatically different moment values. This suggests a different possible explanation than a "Kondo lattice" for the nonmagnetic singlet ground state in materials such as $\mathrm{CeAl}_{3}$, which exhibits a large CF splitting ( $\sim 54 \mathrm{~K})$ and which has been thought ${ }^{41}$ of as the prototype of nonmagnetic Kondo lattices.

\section{CONCLUSION}

In order to understand the main features of the complex phase diagram of $\mathrm{CeSb}$, we have investigated the occurrence of commensurate magnetic structures in which magnetically ordered and non-magnetically-ordered $\{001\}$ planes can coexist. We have used a microscopic model which takes into account two strongly competing mechanisms, the hybridization-mediated two-ion interaction which favors $\langle 001\rangle$ moment alignment and the cubic CF interaction $\left(\Gamma_{7}\right.$ ground state) which favors $\langle 111\rangle$ alignment. We have illustrated that when the two-ion exchange interactions predominate $\left(\Delta_{\mathrm{CF}} \leq E_{1}\right)$, magnetic structures containing only ordered sites are more favorable over the whole temperature range. The tendency to form nonmagnetic planes increases as the ratio $\Delta_{\mathrm{CF}} / E_{1}$ increases, and for sufficiently large $C F$ interaction the magnetic structures containing nonmagnetic planes become the stable phases at high temperatures. The nonmagnetic planes, even though strongly dressed by hybridization, are paramagnetic in nature, in agreement with recent specific heat ${ }^{23}$ and neutron-scattering experiments. ${ }^{19}$ On going to sufficiently low temperature, all cerium sites become magnetically ordered. The presence of strong CF interactions, however, leads to a substantial decrease of the low-temperature ordered moment. This can be counteracted if one introduces weak uniform tetragonal distortion effects which tend to restore the local moment. (The ground-state magnetic structure for the situation summarized by Fig. 2 is type AF-I rather than AF-IA as is experimentally observed. This discrepancy may be due to the omission of the cubic part of the hybridization potential in the treatment leading to the hybridizationmediated anisotropic exchange interactions or may arise from some weak interaction which has not been included in our calculations, such as higher multipolar couplings between cerium ions corresponding to treating the hybridization-induced interactions to higher order in perturbation theory.) Thus the coexistence of magnetically ordered and paramagnetic planes in CeSb is the result of a delicate balance between competing mechanisms, viz., hybridization-mediated interionic exchange, crystal field, and distortional interactions. Furthermore, increasing the crystal-field interaction still further relative to the hybridization-mediated anisotropic interaction, gives rise to a new and interesting low-temperature phenomenon where the system apparently fluctuates between two different states, one of which is a fully nonmagnetic singlet (but not Kondo-like) while the other state has a rather substantial moment. This phenomenon may be pertinent to the behavior of materials such as $\mathrm{CeAl}_{3}$ which have been labeled "Kondo lattices" or "dense Kondo systems."

\section{ACKNOWLEDGMENT}

This research was supported through the National Science Foundation under Grant No. DMR-85-04449. 
*Present address: Department of Physics, California State University-Northridge, Northridge, CA 91330.

†Present address: NASA Lewis Research Center, Cleveland, OH, 44135.

${ }^{1}$ B. R. Cooper and R. Siemann, in Crystalline Electric Field and Structural Effects in f-Electron Systems, edited by J. E. Crow, R. P. Guertin, and T. W. Mihalisin (Plenum, New York, 1980), pp. 141-255.

2J. Rossat-Mignod, P. Burlet, J. Quezel, J. M. Effantin, D. Delacote, H. Bartholin, O. Vogt, and D. Ravot, J. Magn. Magn. Mater. 31-34, 398 (1983).

${ }^{3}$ B. R. Cooper, R. Siemann, D. Yang, P. Thayamballi, and A. Banerjea, in The Handbook on the Physics and Chemistry of the Actinides, edited by A. J. Freeman and G. H. Lander (North-Holland, Amsterdam, 1985), Vol. 2, Chap. 6, pp. 435-500.

${ }^{4}$ G. Busch and O. Vogt, Phys. Lett. 25A, 449 (1967).

${ }^{5}$ B. R. Cooper, M. Landolt, and O. Vogt, in Proceedings of the International Conference on Magnetism ICM-73, edited by $\mathrm{R}$. P. Ozerov and Yu. A. Izyumov (Nauka, Moscow, 1974), Vol. 5, pp. 354-360.

${ }^{6}$ R. J. Birgeneau, E. Bucher, J. P. Maita, L. Passell, and K. C. Turberfield, Phys. Rev. B 8, 5345 (1973).

${ }^{7} \mathrm{~J}$. W. Cable and W. C. Koehler, Magnetism and Magnetic Materials 1971, AIP Conf. Proc. No. 5 (AIP, New York, 1972), p. 1381.

${ }^{8}$ J. Rossat-Mignod, P. Burlet, J. Villain, H. Bartholin, T. S. Wang, D. Florence, and O. Vogt, Phys. Rev. B 16, 440 (1977).

${ }^{9}$ P. Fischer, B. Lebech, G. Meier, B. D. Rainford, and O. Vogt, J. Phys. C 11, 345 (1978).

${ }^{10}$ J. Rossat-Mignod, J. D. Delacote, J. M. Effantin, C. Vettier, and O. Vogt, Physica 120B, 163 (1983).

${ }^{11}$ J. Rossat-Mignod, J. M. Effantin, C. Vettier, and O. Vogt, Physica 130B, 555 (1985).

${ }^{12}$ B. Halg and A. Furrer, J. Appl. Phys. 55, 1860 (1984).

${ }^{13}$ R. Siemann and B. R. Cooper, Phys. Rev. Lett. 44, 1015 (1980); B. R. Cooper, J. Magn. Magn. Mater. 29, 230 (1982).

${ }^{14}$ B. Coqblin and J. R. Schrieffer, Phys. Rev. 185, 847 (1969).

${ }^{15}$ M. A. Rudermann and C. Kittel, Phys. Rev. 96, 99 (1954); T. Kasuya, Prog. Theor. Phys. 16, 45 (1956); 16, 58 (1956); K. Yosida, Phys. Rev. 106, 893 (1957).

${ }^{16}$ B. R. Cooper, G. J. Hu, N. Kioussis, and J. M. Wills, J. Magn. Magn. Mater. 63\&64, 121 (1987); B. R. Cooper, J. LessCommon Metals 133, 31 (1987).

${ }^{17}$ P. Thayamballi, D. Yang, and B. R. Cooper, Phys. Rev. B 29, 4049 (1984).
${ }^{18}$ P. Thayamballi and B. R. Cooper, Phys. Rev. B 31, 5911 (1985).

${ }^{19}$ J. Rossat-Mignod, J. M. Effantin, P. Burlet, T. Chattopadhyay, L. P. Regnault, H. Bartholin, C. Vettier, O. Vogt, D. Ravot, and J. C. Achard, J. Magn. Magn. Mater. 52, 111 (1985).

${ }^{20}$ H. Heer, A. Furrer, W. Halg, and O. Vogt, J. Phys. C 12, 5207 (1979).

${ }^{21}$ M. Escorne, A. Mauger, D. Ravot, and J. C. Achard, J. Phys. C 14, 1821 (1981).

22J. X. Boucherle, A. Delapalme, C. J. Howard, J. RossatMignod, and O. Vogt, Physica 102B, 253 (1980).

${ }^{23}$ J. Rossat-Mignod, P. Burlet, H. Bartholin, O. Vogt, and R. Lagnier, J. Phys. C 13, 6381 (1980).

${ }^{24}$ N. H. Andersen, in Crystalline-Electric Field and Structural Effects in f-Electron Systems, edited by J. E. Crow, R. P. Guertin, and T. W. Mihalisin (Plenum, New York, 1980), pp. 373-387.

${ }^{25}$ W. Selke and M. E. Fisher, Phys. Rev. B 20, 257 (1970).

${ }^{26}$ P. Bak and J. von Boehm, Phys. Rev. B 21, 5297 (1980).

27J. Villain and M. B. Gordon, J. Phys. C 13, 3117 (1980).

${ }^{28}$ H. Takahashi and T. Kasuya, J. Phys. C 18, 2745 (1985).

${ }^{29}$ P. W. Anderson, Phys. Rev. 124, 41 (1961).

30J. R. Schrieffer and P. A. Wolff, Phys. Rev. 149, 491 (1966); J. R. Schrieffer, J. Appl. Phys. 38, 1143 (1967).

${ }^{31}$ J. M. Wills and B. R. Cooper, J. Magn. Magn. Mater. 54-57, 1049 (1986); Phys. Rev. B 36, 3809 (1987).

${ }^{32}$ S. B. Haley and P. Erdos, Phys. Rev. B 5, 1106 (1972); D. H. Yang and Y. L. Wang, Phys. Rev. B 10, 4714 (1974).

${ }^{33} \mathrm{M}$. Tinkham, Group Theory and Quantum Mechanics (McGraw-Hill, New York, 1964).

${ }^{34}$ K. R. Lea, M. J. M. Leask, and W. P. Wolf, J. Phys. Chem. Solids 43, 1381 (1962).

${ }^{35}$ K. W. H. Stevens, Proc. Phys. Soc. London, Sect. A 65, 209 (1952).

${ }^{36}$ B. R. Cooper and O. Vogt, J. Phys. (Paris) Colloq. 32, C1-1026 (1971).

${ }^{37}$ R. Siemann and B. R. Cooper, Phys. Rev. B 19, 2645 (1979).

${ }^{38}$ F. Hulliger, M. Landolt, H. R. Ott, and R. Schmelczer, J. Low-Temp. Phys. 20, 269 (1975).

${ }^{39}$ H. Takahashi, K. Takegahare, A. Yanase, and T. Kasuya, J. Magn. Magn. Mater. 31-34, 405 (1983).

${ }^{40}$ P. Thayamballi and B. R. Cooper, Phys. Rev. B 30, 2931 (1984).

${ }^{41}$ N. B. Brandt and V. V. Moshchalkov, Adv. Phys. 33, 373 (1984). 